INPLASY

PROTOCOL

To cite: Fukuta et al.

Transcatheter interatrial shunt device for the treatment of heart failure with preserved ejection fraction: a protocol for meta-analysis. Inplasy protocol 202120025. doi:

10.37766/inplasy2021.2.0025

Received: 07 February 2021

Published: 07 February 2021

Corresponding author:

Hidekatsu Fukuta

fukuta-h@med.nagoya-cu.ac.jp

Author Affiliation:

Nagoya City University

Graduate School of Medical

Sciences

Support: None.

Review Stage at time of this submission: Preliminary searches.

Conflicts of interest:

None declared.

\section{Transcatheter interatrial shunt device for the treatment of heart failure with preserved ejection fraction: a protocol for meta-analysis}

\author{
Fukuta, $\mathrm{H}^{1}$; Goto, $\mathrm{T}^{2}$; Kamiya, ${ }^{3}$.
}

Review question / Objective: Patients with heart failure with preserved ejection fraction (HFpEF) are often elderly and their primary chronic symptom is severe exercise intolerance. Thus, improvement of exercise capacity presents an important clinical outcome in HFpEF patients. A steep increase in left atrial pressure during exercise is an important cause of exertional dyspnea in HFpEF patients. The creation of a left-to-right interatrial shunt to decompress the left atrium may improve symptoms in patients with HFpEF. A novel transcatheter interatrial shunt device has been developed and evaluated in patients with HFpEF in several clinical trials. However, there is insufficient evidence regarding the benefit of transcatheter interatrial shunt device in these patients. The purpose of this meta-analysis is to evaluate the efficacy as well as safety of transcatheter interatrial shunt device in HFpEF patients compared with standard therapy.

Condition being studied: Patients who are symptomatic despite optimal guideline-directed medical therapy.

INPLASY registration number: This protocol was registered with the International Platform of Registered Systematic Review and Meta-Analysis Protocols (INPLASY) on 07 February 2021 and was last updated on 07 February 2021 (registration number INPLASY202120025).

\section{INTRODUCTION}

Review question / Objective: Patients with heart failure with preserved ejection fraction (HFpEF) are often elderly and their primary chronic symptom is severe exercise intolerance. Thus, improvement of exercise capacity presents an important clinical outcome in HFpEF patients. A steep increase in left atrial pressure during exercise is an important cause of exertional dyspnea in HFpEF patients. The creation of a left-to-right interatrial shunt to decompress the left atrium may improve symptoms in patients with HFpEF. A novel transcatheter interatrial shunt device has been developed and evaluated in patients with HFpEF in several clinical trials. 
However, there is insufficient evidence regarding the benefit of transcatheter interatrial shunt device in these patients. The purpose of this meta-analysis is to evaluate the efficacy as well as safety of transcatheter interatrial shunt device in HFpEF patients compared with standard therapy.

Condition being studied: Patients who are symptomatic despite optimal guidelinedirected medical therapy.

\section{METHODS}

Participant or population: Patients with heart failure with preserved ejection fraction.

Intervention: Transcatheter interatrial shunt device.

Comparator: Patients who are on optimal guideline-directed medical therapy.

Study designs to be included: Randomized controlled trials (RCTs) and single-arm trials.

Eligibility criteria: This meta-analysis will include: (1) trials including patients with HFpEF; (2) RCTs or single-arm trials; (3) used transcatheter interatrial shunt device; and (4) assessed exercise capacity, symptoms, quality of life, morbidity, or mortality.

Information sources: PubMed, Web of Science, Cochrane Library, and ClinicalTrials.gov .

Main outcome(s): Exercise capacity (6minute walking distance).

Additional outcome(s): Heart failure symptoms, health-related quality of life, and mortality and hospitalization rates.

Quality assessment / Risk of bias analysis: The Cochrane Risk of Bias tool will be used to assess quality of RCTs included. The quality of evidence for the outcomes will be evaluated by use of the Grading of Recommendations Assessment,
Development and Evaluation (GRADE) system. The quality of evidence will be evaluated across the domains of risk of bias, consistency, directness, precision, and publication bias.

Strategy of data synthesis: For continuous outcomes, weighted mean differences and corresponding $95 \%$ confidence interval will be calculated. For dichotomous outcomes, relative risks with $95 \%$ confidence interval will be calculated. For each outcome, heterogeneity will be assessed using the Cochran's $Q$ and 12 statistic; for the Cochran's $Q$ and 12 statistic, a $p$ value of $50 \%$, will be considered significant, respectively. When there is significant heterogeneity, the data will be pooled using a random-effects model, otherwise a fixedeffects model will be used. Publication bias will be assessed graphically using a funnel plot and mathematically using Egger test. For these analyses, Comprehensive Meta Analysis Software version 2 (Biostat, Englewood, NJ, USA) and STATA 16 software (Stata Corp LP, TX, USA) will be used.

Subgroup analysis: Meta-analyses will be performed separately for randomized controlled trials and single-arm trials.

Sensitivity analysis: Meta-regression will be used to determine whether the effect of transcatheter interatrial shunt device will be confounded by baseline clinical characteristics.

Language: Only articles published in the English language will be included.

Country(ies) involved: Japan.

Keywords: interatrial shunt, heart failure, meta-analysis.

Contributions of each author:

Author 1 - Hidekatsu Fukuta.

Email: fukuta-h@med.nagoya-cu.ac.jp

Author 2 - Toshihiko Goto.

Email: t-goto@med.nagoya-cu.ac.jp

Author 3 - Takeshi Kamiya.

Email: kamitake@med.nagoya-cu.ac.jp 